\title{
A Vigilância Epidemiológica e a Infecção pelo HIV
}

\author{
Marilia Sá Carvalho*
}

Este artigo discute três métodos de obtenção da informaçấo necessária à vigilância epidemiológica da AIDS: inquéritos soroepidemiológicos em grupos populacionais determinados, utilização de serviços-sentinela e notificaçáo compulsória dos soropositivos. De acordo com a experiência da equipe do Programa de Prevençáo e Controle da AIDS da Secretaria Estatual de Saúde do Rio de Janeiro, quase toda a informação existente atualmente baseia-se na notificação de casos confirmados da doença. No sentido de monitorar os niveis e tendências da infecção pelo HIV , este trabalho sugere a utilização dos três métodos de vigilância discutidos, garantindo a maior efetividade do programa de prevenção e controle de AIDS.

\section{INTRODUÇÃO}

Desde 1982, quando foi diagnosticado o primeiro caso no país, até novembro de 1988 , foram registrados 4946 casos confirmados de AIDS, dos quais 752 no Rio de Janeiro (MS, 1988a). Nestes quase sete anos, foram observadas algumas mudanças no padrão de transmissão da doença. Destacam-se, como mais importantes (Castilho, 1988):

- a diminuição do predomínio de casos no sexo masculino, sendo que a relação entrehomens/mulheres atingidos passou de 28:1 em 1986 para 7:1 em final de 88 (MS, 1988b);

- o pequeno aumento relativo da transmissão sanguínea como um todo, havendo entretantoincremento substancial entre os usuários de drogas;

- o aumento relativo de casos por transmissão heterossexual.

É fundamental colocar em discussão a qualidade e a pertinência da informação disponível quando se analisa o padrāo epidemiológico da doença e, principalmente, para garantir o desenvolvimento de um efetivo

*Pesquisadora auxiliar do Departamento de Epidemiologia $e$ Métodos quantitativos em Saúde, ENSP. programa de prevenção e controle. Este é objetivo deste trabalho, que surgiu a partir de discussóes com os técnicos do Programa de Prevenção e Controle de AIDS da Secretaria de Estado de Saúde do Rio de

Cadernos de Saúde Pública, RJ, 5 (2): 160-168, abr/jun, 1989 
janeiro. Por este motivo, praticamente a totalidade dos exemplos e situações colocadas no texto se referem à realidade do Rio de Janeiro, embora acreditemos que a situação de outros estados não seja muito diferente.

\section{A QUALIDADE DA INFORMAÇÃO EPIDEMIOLÓ- GICA SOBRE A AIDS}

A tualmente, por norma do Ministério da Saúde, é obrigatória a notificação apenas de casos confirmados de AIDS, definidos como:

"a) todo indivíduo que apresente doença pelo menos moderadamente indicativa de imunodeficiência celular, segundo critérios estabelecidos (...) excluídas outras causas (...)

b) todo indivíduo que evoluiu para óbito de causa natural não esclarecida suficientemente para exclusão de doença associada a infecção pelo HIV e que apresentava o seguinte conjunto de condiçōes: - soropositividade para o HIV; - presença de candidíase oral e ou testes cutâneos de hipersensibilidade tardia negativos; - presença de três ou mais dos seguintes sintomas (...); - ausência de outras causas sabidamente associadas a diminuição da resistência, que não a infecção pelo HIV." (MS, 1988c)

As mesmas normas são utilizadas no Estado do Rio de Janeiro, onde foi adotada a seguinte rotina de funcionamen ${ }^{+} o$ ào sistema de vigilância epidemiológica:

- todos os casos notificados (e só é compulsória a notificação dos casos confirmados) são investigados pelo nivel central da Secretaria Estadual de Saúde ou pela Secretaria municipal de Saúde do Rio de Janeiro, município onde se concentra a maioria dos pacientes;

- esta investigação é feita através do preenchimento de uma ficha epidemiológica com dados levantados no prontuário do paciente;

- os principais objetivos da investigação epidemiológica são a confirmaçâo do caso, observando os critérios definidos acima e registrando a manifestação clínica que selou o diagnóstico e a verificação do mecanismo de transmissăo do vírus e dos fatores de risco; - os óbitos possivelmente causados pela AIDS também são notificados e investigados.

Algumas conseqüências desta rotina na qualidade e oportunidade da informação são evidentes. Em primeiro lugar, a centralizaçắo de toda a investigação, se no início da epidemia era justificável, por ser uma doença nova e com pequeno número de casos, atualmente representa um enorme entrave à obtenção de informaçâo. 
Outro aspecto prejudicial desta centralização, é que qualquer problema conjuntural que afete uma destas duas instituições acarreta atrasos consideráveis na obtenção da informação. São bons exemplos disto a recente greve da Secretaria Municipal de Saúde, com duração superior a cinco meses, e a carência conjuntural de pessoal no programa de AIDS da Secretaria Estadual de Saúde.

As consequiências numéricas deste fato eram, em dezembro de 88 :

- 346 óbitos notificados e ainda não investigados no município do Rio de Janeiro;

- 134 casos notificados no interior do estado e ainda nâo investigados;

- um total de aproximadamente 900 casos e óbitos sem investigação.

Também a qualidade da investigação epidemiológica está longe de ser razoável. O processo de coleta da informação baseia-se nos dados disponíveis nos prontuários, copiados por técnico especialmente designado para esta atividade, que em geral não trabalha no serviço.Infelizmente não se pode garantir a qualidade de preenchimento dos prontuários nos serviços de saúde brasileiros. Além disto, diversas informaçōes relativas a, por exemplo, prática sexual e utilização de drogas, entre outras, como não dizem respeito às condiçōes de saúde imediatas de cada paciente, são freqüentemente relegadas a segundo plano. A conseqüêencia imediata disto é 0 alto percentual de casos sem definiçāo do (s) mecanismo (s) de transmissāo evolvido (s). No Brasil, este percentual está em torno de $7,5 \%$ (MS, 1988a) e no Estado do Rio de Janeiro de $13,6 \%$ (SES, 1988).

Merece ainda destaque na análise da qualidade das informaçōes sobre a epidemiologia da AIDS no país a dificuldade de se obter informaçōes completas sobre a evolução clínica dos pacientes. Graças à permanente superlotação dos principais hospitais que dispõem de um maior número de leitos específicos para a doença, aliada à dificuldade de garantir o atendimento em hospitais gerais, a rotatividade dos pacientes é generalizada. Os aidéticos, em sua maioria, sāo atendidos em diferentes locais no decurso da doença, dificultando mui to os estudos epidemiológicos de incidência de infecçōes oportunistas. Estes têm grande importância pois a evoluçāo clínica da doença sofre influência decisiva das doenças infecciosas predominantes no país. Embora este problema não esteja diretamente relacionado à vigilância epidemiológica, suas consequeências a médio prazo são vitais na análise da epidemiologia da AIDS, e de outras doenças que possam ter sua tendência modificada pela associação com o HIV. 


\section{A INFECÇÃO PELO HIV}

Toda informação disponível, de melhor ou pior qualidade, é sobre o doente de AIDS, refletindo um padrão de transmissão de aproximadamente cinco anos atrás. Inquéritos especiais de soropositividade realizados em alguns grupos populacionais específicos sugerem cifras variadíssimas: de $0 \%$ a $25 \%$ entre prostitutas; de $19 \%$ a $36 \%$ entre homossexuais; de $20 \%$ a $76 \%$ entre hemofilicos. (Castilho, 1988)

Mesmo a informação da prevalência da soropositividade em doadores de sangue, usualmente aceita como um bom estimador da prevalência na população em geral, varia entre $0,03 \%$ e $0,7 \%$. (Castilho, 1988) Além disso, estes resultados certamente so frem distorção de eventos circunstanciais, como por exemplo, a exclusão mais ou menos rigorosa de pessoas que apresentam fatores de risco, ou, no sentido inverso, a procura dos bancos de sangue por pessoas desejosas de serem gratuitamente testadas para a doença, na inexistência de acesso a atendimento para tal.

Por outro lado, a necessidade de informação fez com que fosse colocado, pela OMS, como elementochave dos programas nacionais de prevenção e controle da AIDS a seguinte recomendação:

"... estabelecimento de um sistema de vigilância da AIDS e, sobretudo, da infecção pelo HIV" (OMS, 1988).

O acompanhamento da tendência da infecção pelo HIV é vital para:

- estimar a prevalência da doença no futuro próximo $e$, conseqüentemente, viabilizar o planejamento do atendimento médico, laboratorial e hospitalar aos doentes, facilitando técnica e politicamente a alocação de recursos para tal;

- acompanhar a evolução no padrão de transmissão do HIV, potencializando os programas educativos, dirigindo-os aos grupos em maior risco e aos comportamentos preferenciais na disseminação da doença;

- avaliar o impacto do conjunto de medidas de prevenção adotadas pelos diversos órgãos e entidades envolvidos.

Diversos métodos são necessários para que se estabeleça um quadro, o mais aproximado possível da realidade, sendo que nos deteremos em três deles nó momento: inquéritos especiais de soroprevalência, serviços-sentinela e notificação compulsória dos indivíduos soropositivos.

\section{INQUÉRITOS SOROEPIDEMIOLÓGICOS}

Utilizados em diversas doenças transmissiveis, os inquéritos epidemiológicos de prevalência realizados 
a partir de testes imunológicos têm inegáveis méritos: - amostra aleatória de grupos populacionais onde se deseja estimar a prevalência da infecção, evitando assim os vieses de seleção, e aumentando a representatividade dos resultados em relação a toda população de origem;

- controle do viés introduzido por auto-seleção, ou recusa, desde que níveis aceitáveis de não-respostas; - seleção da população onde se pretende estimar a prevalência, permitindo a avaliaçáo posterior de programas de prevenção desenvolvidos junto às populações estudadas.

Alguns problemas entretanto merecem atenção. Em primeiro lugar, estes estudos são de altíssimo custo para nossa realidade. Por um lado, o custo do próprio exame, até hoje escasso até para a população interessada voluntariamente em realizá-lo, e, mais importante, o custo operacional destes estudos, envolvendo desde entrevistadores e técnicos de laboratório, até pessoal altamente especializado para o desenho da pesquisa e análise dos resultados.

Outro problema, de cunho ético, está na necessidade de esclarecer ao entrevistado o motivo da pesquisa, obtendo sua plena concordância. Evidentemente, qualquer recusa nestas circustâncias pode ter relação com o objetivo da pesquisa, introduzindo exatamente o problema que se queria evitar: a auto-exclusão.

Diversos métodos vêm sendo testados em outros países buscando-se a superação destes problemas (OMS, 1988). Por exemplo, estudos cegos, onde o sangue de indivíduos colhidos para outra finalidade, é eliminada toda identificação, e os soros são testados anonimamente.

Este é apenas um exemplo de estratégias que podem e precisam ser buscadas no sentido de se conhecer a distribuição dos soropositivos. Este tipo de pesquisa, embora de interesse primordial para os serviços de saúde pelas informaçōes que fornece quanto aos rumos da epidemia, é mais facilmente executada pelas instituiçôes acadêmica e de pesquisa interessadas em trabalhar com a doença.

\section{Serviços-Sentinela}

O acompanhamento de tendências de incidência e a detecção precoce de surtos são freqüentemente viabilizadas através das chamadas unidades-sentinela. Estas nada mais são que serviços de saúde que, por sua localização ou pelas características de seu atendimento, têm maior probabilidade de entrar em contato com os casos.

Em relação a detectar os níveis e, principalmente, as tendências da infecção pelo HIV , clínicas de aconse- 
lhamento e realização do teste sorológico seriam elementos-chave que facilitariam vir à tona um expressivo número soropositivos assintomáticos. A investigação destes em relação a modos de transmissão e comportamentos de risco traria elementos valiosos na compreensão do processo de infecção pelo HIV. Além, evidentemente dos benefícios trazidos aos próprios, que receberiam apoio psicológico e orientação em relaçāo a fatores de risco adicionais no desencadeamento das manifestações clínicas e quanto à transmissibilidade a outras pessoas.

$\mathrm{Na}$ situação atual, em função da limitada capacidade dos serviços de saúde que atendem a AIDS, do tempo de espera e da restrição de exames, apenas um número inexpressivo de soropositivos é atendido. Mesmo a prática usual de alguns hospitais de chamar os comunicantes dos casos assistidos para realização de teste sorológico e aconselhamento vem sendo abandonada devido à sobrecarga dos serviços.

A vigilância epidemiológica da infecção pelo HIV com a utilização deste tipo de sentinelas tem algumas limitações:

- os testes serão feitos em população auto-selecionada, com padrōes de infecção e taxas de prevalência $e$ incidência provavelmente muito maiores do que as da população em geral;

- a necessidade de garantir o anonimato no exame, o que pode dificultar a análise das informações.

\section{Notificação Compulsória dos Soropositivos}

As limitações que a notificação de rotina têm são sobejamente conhecidas: falta de interesse dos profissionais em notificar, erros e atrasos na notificação, qualidade precária das informações coletadas, entre outras. A estas são acrescentados outros problemas específicos para a AIDS:

- temor de que mesmo antes do desenvolvimento de qualquer doença o soropositivo assintomático venha a sofrer cerceamentos e restrições prejudiciais à sua vida;

- receio de que esta medida preceda a obrigatoriedade de testes sorológicos em diversas situaçōes da vida pessoal e profissional dos indivíduos;

- medo de que esta informação seja tratada de forma irresponsável, causando prejuízos aos indivíduos;

- o conjunto destas questôes contribuindo para tornar clandestinos os exames sorológicos.

É exatamente este tipo de questão que fez com que, por exemplo nos Estados Unidos, 11 estados tenham tornado obrigatória a notificação dos soropositivos, enquanto os demais contam apenas com a notifi- 
cação de casos clínicos. (Dondero, 1988) É evidente que apenas tomar obrigatória a notificação não trará toda a informação necessária ao acompanhamento da tendência da infecção pelo HIV. Entretanto este é um recurso a mais, especialmente importante na nossa realidade, onde dificilmente se fará inquéritos populacionais de rotina, nos quais se baseia, por exemplo, a vigilância epidemiológica da infecção pelo HIV nos EEUU.

Entretanto, esta notificação deveria ser cercada de medidas especiais, que garantissem o sigilo das informações, e aumentassem a confiabilidade dos serviços de saúde, especialmente os públicos. Além disso, seria importante garantir que:

- nas clínicas de aconselhamento os testes pudessem ser realizados anonimamente, se assim o desejasse o paciente;

- todos os casos notificados entrariam no sistema de informação, sendo investigados;

- os laboratórios e médicos particulares também fomecessem informações;

- a informação produzida fosse devolvida aos informantes, como forma de manter o interesse e estimular a notificação.

No Estado do Rio de Janeiro a experiência já acumulada com a notificação compulsória dos soropositivos detectados entre os doadores de sangue até o momento não trouxe problemas de quebra de sigilo, ou outros prejuizos. É importante observar, que todos os soros positivos com o ELISA, são encaminhados ao nível central da Secretaria Estadual de Saúde, que encaminha aerograma solicitando o comparecimento dos doadores para novos exames, buscando a confirmação da positividade através de outra técnica laboratorial. Em que pesem as limitações do processo, tais como demora na entrega dos resultados, do próprio aerograma como meio de contato com os indivíduos é, evidentemente, positiva a norma estabelecida no sentido da confirmação da soropositividade através da realização de outros testes.

Algumas experiências como, por exemplo, a desenvolvida no Estado do Colorado/EEUU, (Judson, 1988) sugerem que, dadas as devidas garantias, éticas e legais, de respeito aos direitos do cidadão, a obrigatoriedade da notificação não aumenta o estigma que pesa sobre os portadores do HIV, e traz importantes informações sobre as tendências da infecção pelo vírus.

\section{COMENTÁRIOS FINAIS}

Desenvolver o conhecimento sobre a AIDS é uma necessidade vital na busca da prevenção da doença. 
São necessários conhecimentos sobre comportamentos de risco que possam ter influência na disseminação do HIV; sobre impacto das medidas educativas na alteração de comportamentos; sobre a influência do HIV na epidemiologia de outras doenças prevalentes no país e vice-versa. A vigilância epidemiológica é um meio indispensável na produção de informação. (Judson, 1988)

Os três métodos de obtenção de dados para estudar as tendências da infecção pelo HIV são indispensáveis e complementares, e só poderão ser desenvolvidos com um esforço multiinstitucional, envolvendo secretarias de saúde, em seus vários serviços, universidades, instituiçōes de pesquisa, e comunidade interessada.

Outro aspecto fundamental a ser considerado é a qualidade da informação e sua utilização. A notificação do soropositivo só faz sentido se cada caso notificado for devidamente investigado, no sentido de se produzir informação confiável sobre comportamentos de risco, mecanismos de transmissão, entre outras. Além disto, a divulgação da informação produzida deve ser um compromisso fundamental das instituiçōes. Baseados nela os dirigentes dos órgãos públicos poderão estabelecer políticas de prevenção mais eficazes e os produtores de dados verificaráo a importância de seu trabalho. Além disso, é a informação clara e objetiva que permitirá à população acompanhar 0 que é feito, e para onde caminha a doença, cobrando sempre que necessário as medidas que julgar corretas.

\section{AGRADECIMENTOS}

Este trabalho só foi possível graças aos técnicos do Programa de Prevençấo e Controle da AIDS, da Secretaria Estadual de Saúde do Rio de Janeiro, que além de permitirem a utilização dos dados, participaram de diversas discussões de avaliação do programa, onde foram sendo ellaboradas as linhas gerais das opiniöes expressadas no artigo. Em especial, agradecimentos para Álvaro Matida, coordenador do programa, Regina Guedes de Carvalho, Kátia Regina Valente de Lemos e Joaquim Valente.

This paper discusses three methods for obtaining information for the epidemiological surveillance of AIDS: seroepidemiological surveys among selected population groups sentinel surveillance approaches and compulsory notification of seropositives. As the 
experience of the AIDS Programme team of the State Secretariate of Health, Rio de Janeiro has shown. almost all available information focusses on the notification of confirmed cases. In attempt to monitor the levels and trends of HIV infection, this paper suggests that the three methods of surveillance should be used to ensure greater effectiveness in the prevention and control of AIDS.

\section{REFERÊNCIAS BIBLIOGRÁFICAS}

CASTILHO, Euclides C. Padräo Epidemiológico da AIDS no Brasil. Boletim epidemiológico AIDS, ano Il, $n: 2,1988$.

CURRAN, J.W., Allen, J.R.: Prevention of AIDS and HIV Infection: Needs and Priorities for Epidemiologic Reserarch. Am $J$ Public Health 78(4): 381-386, april 1988.

DONDERO, T.J., Pappaioanou, M., Curran, J. W.: Monitoring the Levels and Trends of HIV Infection: the Public Health Servive's HIV Surveillance Program. Public Health Rep 103 (3): 213-220, May-June 1988.

JUDSON, F. N., Vermon, T. M.: The impact of AIDS on State and Local Health Departments: Issues and a Few Answers. Am J Public Health 78 (4): 387-393, April 1988.

Ministério da Saúde Boletim Epidemiológico AIDS, ano II, n:5, tabela II, 1988.

MINISTÉRIO DA SAÚDE BOLETIM EPIDEMIOLÓGICO AIDS, ano II, ediçąo especial, gráfico 3, dez. 1988.

MINISTÉRIO DA SAÚDE - SNABS. Programa Nacional De Controle de Doenças Sexualmente Transmissiveis e AIDS. Recomendações para a prevenção e controle da infecção pelo vírus HIV (SIDA-AIDS). - Brasília: Centro de Documentação do Ministério da Saúde, Série A: Normas e Manuais Técnicos, 42, 1987.

OMS. Programa Global de SIDA-AIDS. Ação em SIDA-AIDS. n: 1 , p. 5, agosto/1988.

SECRETARIA ESTADUAL DE SAÚDE. Superintendência de Saúde Coletiva. Departamento de Doenças Transmissíveis. Programa de Prevençāo e Controle de DST-AIIDS. Dados de outubro de 1988, Rio de Janeiro. 